\title{
Search strategies for top partners in composite Higgs models
}

\author{
Ben Gripaios, Thibaut Müller, M.A. Parker and Dave Sutherland \\ Cavendish Laboratory, \\ J.J. Thomson Avenue, Cambridge, U.K. \\ E-mail: gripaios@hep.phy.cam.ac.uk, mueller@hep.phy.cam.ac.uk, \\ parker@hep.phy.cam.ac.uk, dws28@cam.ac.uk
}

ABSTRACT: We consider how best to search for top partners in generic composite Higgs models. We begin by classifying the possible group representations carried by top partners in models with and without a custodial $\mathrm{SU}(2) \times \mathrm{SU}(2) \rtimes \mathbb{Z}_{2}$ symmetry protecting the rate for $Z \rightarrow b \bar{b}$ decays. We identify a number of minimal models whose top partners only have electric charges of $\frac{1}{3}, \frac{2}{3}$, or $\frac{4}{3}$ and thus decay to top or bottom quarks via a single Higgs or electroweak gauge boson. We develop an inclusive search for these based on a top veto, which we find to be more effective than existing searches. Less minimal models feature light states that can be sought in final states with like-sign leptons and so we find that 2 straightforward LHC searches give a reasonable coverage of the gamut of composite Higgs models.

KEywords: Phenomenological Models, Hadronic Colliders

ARXIV EPRINT: 1406.5957 


\section{Contents}

1 Introduction 1

2 Taxonomy of top partners $\quad 3$

3 Phenomenology of the singlet, doublet and triplet models 4

3.1 Singlet model 4

3.2 Doublet model 5

3.3 Triplet models 6

$\begin{array}{ll}3.4 & \text { Larger representation models }\end{array}$

4 Current experimental limits on the singlet and doublet models $\quad 8$

4.1 SUSY searches 8

4.2 Dedicated searches 8

$\begin{array}{lll}5 & \text { New analysis design } & 8\end{array}$

6 Limits on the singlet and doublet models 11

$\begin{array}{lll}7 & \text { Discussion } & 11\end{array}$

$\begin{array}{ll}\text { A Group and representation theory of top partners } & 13\end{array}$

B Details of minimal model without $Z \rightarrow b \bar{b}$ protection $\quad 18$

\section{Introduction}

Models in which the naturalness problem of the Standard Model (SM) is solved by making the Higgs a composite, pseudo-Nambu-Goldstone boson (PNGB) of some as-yet-unknown strong dynamics [1-3] have come to the fore in recent years as they have matured [4-6], incorporating symmetries [7, 8] and partial compositeness [9] to mitigate unobserved corrections in electroweak precision tests and flavour physics. They are, by now, arguably just as good (or just as bad) a contender as supersymmetry for new physics at the $\mathrm{TeV}$ scale, and the LHC experiments ought to devote significant resources to looking for them.

Just as for supersymmetry, the composite Higgs is more a paradigm than a model, in that it may be realised in arbitrarily many ways. Thus it is not clear, a priori, how best to search at the LHC for evidence of a composite Higgs. Naïvely, the obvious place to look is in the Higgs sector itself, but the minimal model [5] contains just a single SM Higgs doublet (for models with an extended Higgs sector, see, e.g. [10, 11]). Moreover, the apparent absence of new physics in the electroweak and flavour sectors already forces us 
into a slightly-tuned regime in which the electroweak scale is suppressed compared to the scale of strong dynamics, which suggests that the couplings of a composite Higgs to other SM particles will deviate by only a few percent from the SM values $[12,13]$.

The necessary suppression also implies that new states, beyond the SM, will generically be out of the mass reach of the LHC. However, the mechanism of partial compositeness requires the existence of additional states that mix with the top quark and several authors have argued [14-20] that these "top partners" should be light, given the measured Higgs boson mass. In a nutshell, the connection arises because the Higgs potential (and, ergo, the Higgs mass) is predominantly induced by radiative corrections involving the top quark, which are cut off by the top partners.

Many suggestions for dedicated searches for such top partners have been put forward [21-38]. Indeed, there are already more on the market than the experiments have the resources to implement, with just a few analyses having appeared thus far.

We thus consider the question of whether the experiments could obtain a reasonable coverage of the space of composite Higgs models with just a small number of generic searches. To do so, we must first classify the possible top partners and their phenomenological implications. In all the models that have appeared so far, the top partners are colour triplets, just like the top quark, ${ }^{1}$ and so can be pair-produced at the LHC via strong interactions with significant cross-section. ${ }^{2}$ It is the subsequent decays that give us the most phenomenological room to manœuvre. The possible decays are, of course, restricted by the electric charge, which must equal $\pm \frac{2}{3}$ modulo any integer, and we shall see below that any such charge is possible for the lightest top partner.

If the lightest states have charge not equal to $\frac{1}{3}, \frac{2}{3}$, or $\frac{4}{3}$, then they decay via multiple $W$ bosons and lead to easily identifiable final states with like-sign leptons [21]. Other states pose more of a problem for experimental searches, since they can decay to a variety of states ( $W q, Z q$, or $H q$, where $q$ is a top or bottom quark) with comparable branching fractions at most points in parameter space. We find that, rather than developing specific searches targeted towards the different decay channels, it is more effective to simply reduce the dominant SM backgrounds (especially $t \bar{t}$ ) by vetoing events with a top quark, in a certain way.

The outline is as follows. In the next section, we begin with a group-theoretic classification of possible top partners. We show that in general it is possible to have a lightest top partner with any electric charge, but that there are restrictions if we ask that the theory have a symmetry protecting the decay rate for $Z \rightarrow b \bar{b}[8]$. Restrictions also arise if we insist that the right-handed top, $t_{R}$, be wholly composite. The results will, no doubt, come as no surprise to experts, but we have not been able to find them in the literature. In section 3, we discuss the minimal models that only contain top partners with charge $\frac{1}{3}, \frac{2}{3}$, or $\frac{4}{3}$. These have top partners in 1-, 2-, and 3-dimensional representations, and there are 2 inequivalent representations of each dimension, giving 6 models in all. The 1-d models (discussed already in [29]) allow for both custodial protection of $Z \rightarrow b \bar{b}$ and

\footnotetext{
${ }^{1}$ Models in which the lightest top partners are not colour triplets are conceivable, but will typically feature leptoquark states [39], which themselves provide an alternative target for LHC searches [40].

${ }^{2}$ There may also be a significant cross-section for single production, in association with quarks.
} 
$t_{R}$ compositeness. The other models allow neither, but are of interest as straw models, as their phenomenology is rather different. In the doublet and triplet models, the lightest states have charges $\frac{1}{3}$ and $\frac{4}{3}$, respectively. We also give an explicit effective Lagrangian description for the doublet models, following the approach pioneered for the 1-d models in [29]. In sections $4-5$, we estimate the limits on these models that are obtained using a slew of existing LHC searches and show how a single search based on a top veto is more effective in all cases.

\section{Taxonomy of top partners}

We first wish to classify the possible top partners, which requires us to discuss their representations (henceforth 'reps') and in turn the groups whose reps they carry. Since we wish to include in our discussion the custodial symmetry for $Z \rightarrow b \bar{b}$, it will not suffice to discuss Lie algebras; we must discuss Lie groups. We give only the salient definitions and results here, relegating most of the details and proofs to appendix A.

A composite Higgs model is based on a coset $G / H$, and the usual rules of non-linear sigma models [41] dictate that the top partner must carry a rep of $H$. As we argue in appendix A, it suffices to consider $G=\mathrm{Sp}(2)$ and either $H=\mathrm{SU}(2) \times \mathrm{SU}(2)$ (henceforth denoted $\left.\mathrm{SU}(2)^{2}\right)$ or $H=\mathrm{SU}(2)^{2} \rtimes \mathbb{Z}_{2}$, depending on whether we wish to have custody of $Z \rightarrow b \bar{b}$ or not. In the semi-direct product in $\mathrm{SU}(2)^{2} \rtimes \mathbb{Z}_{2}$, the $\mathbb{Z}_{2}$ is mapped to the automorphisms of $\mathrm{SU}(2)^{2}$ given by permutations of the $2 \mathrm{SU}(2)$ subgroups.

The unitary irreducible reps (irreps) of $\operatorname{Sp}(2)$ can be labelled by $(m, k)$, where $m \geq$ $k \geq 0$ and $2 m+1$ and $2 k+1$ are either both even or both odd, and have dimension $(2 k+1)(2 m+3)(m+k+2)(m-k+1) / 6$. The irreps of $\mathrm{SU}(2)^{2}$ can be labelled by $(2 l+1,2 r+1)$, where $2 l+1 \in \mathbb{N}$ denotes the dimension of the spin- $l$ irrep of SU(2) (and similarly for $r$ ), and have dimension $(2 l+1)(2 r+1)$. The irreps of $\mathrm{SU}(2)^{2} \rtimes \mathbb{Z}_{2}$ fall into 3 classes, which we label by $(2 l+1,2 l+1)^{ \pm}$and $((2 l+1,2 r+1))$, of dimensions $(2 l+1)^{2}$ and $2(2 l+1)(2 r+1)$, respectively. The branching rules for $\mathrm{Sp}(2) \rightarrow \mathrm{SU}(2)^{2} \rtimes \mathbb{Z}_{2}$ are given in appendix $\mathrm{A}$, and are shown explicitly in table 1 for dimensions up to 50 . The branching rules for $\mathrm{SU}(2)^{2} \rtimes \mathbb{Z}_{2} \rightarrow \mathrm{SU}(2)^{2}$ are $(2 l+1,2 l+1)^{ \pm} \rightarrow(2 l+1,2 l+1)$ and $((2 l+1,2 r+1)) \rightarrow(2 l+1,2 r+1) \oplus(2 r+1,2 l+1)$.

The classification of top partners is then as follows. If we desire custody of $Z \rightarrow b \bar{b}$, the top partner must carry one of the irreps $(2 l+1,2 l+1)^{ \pm}$or the irrep $((2 l+1,2 l+3))$, for some $l$. If we desire a fully composite $t_{R}$, then the top partner must carry one of the irreps $(2 l+1,2 l+1)^{ \pm}$of $\mathrm{SU}(2)^{2} \rtimes \mathbb{Z}_{2}$ (or $(2 l+1,2 l+1)$ of $\left.\mathrm{SU}(2)^{2}\right)$. If we desire neither, the top partner may carry any irrep of $\mathrm{SU}(2)^{2}$.

For each of these top partner reps, we are still left with an infinity of possible models, because we are free to assign the elementary $Q_{L}$ to a spurionic irrep of $\operatorname{Sp}(2)$ with arbitrarily large $2 m+1$. For example, in a model with a top partner carrying either the $(1,1)^{+}$or $(1,1)^{-}$, respectively, of $\mathrm{SU}(2)^{2} \rtimes \mathbb{Z}_{2}$, the $Q_{L}$ can be assigned to any irrep $(m, 0)$ of $\operatorname{Sp}(2)$ with $m$ even or odd, respectively.

Models with top partners carrying either the $(1,1)$ (or $\left.(1,1)^{ \pm}\right),(2,1)$ (or $\left.(1,2)\right)$, or $(3,1)$ (or $(1,3)$ ) irreps pose a particular challenge phenomenologically, since these can 


\begin{tabular}{|ccc|}
\hline $\mathrm{Sp}(2)$ irrep $(m, k)$ & dimension & $\mathrm{SU}(2)^{2} \rtimes \mathbb{Z}_{2}$ irreps \\
\hline$(0,0)$ & 1 & $(1,1)^{+}$ \\
$\left(\frac{1}{2}, \frac{1}{2}\right)$ & 4 & $((2,1))$ \\
$(1,0)$ & 5 & $(1,1)^{-} \oplus(2,2)^{-}$ \\
$(1,1)$ & 10 & $((3,1)) \oplus(2,2)^{+}$ \\
$(2,0)$ & 14 & $(1,1)^{+} \oplus(2,2)^{+} \oplus(3,3)^{+}$ \\
$\left(\frac{3}{2}, \frac{1}{2}\right)$ & 16 & $((2,1)) \oplus((3,2))$ \\
$\left(\frac{3}{2}, \frac{3}{2}\right)$ & 20 & $((4,1)) \oplus((3,2))$ \\
$(3,0)$ & 30 & $((5,1)) \oplus((4,2)) \oplus(3,3)^{+}$ \\
$(2,2)$ & 35 & $(2,2)^{-} \oplus(3,3)^{-} \oplus(4,4)^{-}$ \\
$(2,1)$ & 35 & $((2,1)) \oplus(2,2)^{-} \oplus((4,2)) \oplus(3,3)^{-}$ \\
$\left(\frac{5}{2}, \frac{1}{2}\right)$ & 40 & $((3,2)) \oplus((4,3))$ \\
\hline
\end{tabular}

Table 1. $\mathrm{Sp}(2) \rightarrow \mathrm{SU}(2)^{2} \rtimes \mathbb{Z}_{2}$ branching rules for dimensions up to 50 .

feature only states with charges $\frac{1}{3}, \frac{2}{3}$, or $\frac{4}{3}$. In what follows, we consider the prospects for experimental searches in these worst-case scenarios. To get a reasonable idea of the limits that can be obtained, it suffices to consider just one model for each possible dimension of the top partner irrep. Other models (including those with different choices of the $\mathrm{Sp}(2)$ irrep) have the same spectrum of states, but with varying branching ratios. Since our proposed search includes all decay modes, the limits obtained should not vary too significantly.

\section{Phenomenology of the singlet, doublet and triplet models}

In what follows, we will only discuss experimental searches for 3 of the minimal models just described, namely a $(1,1)^{-}$model containing only a $T$ (charge $\left.\frac{2}{3}\right)$; a $(1,2)$ model containing a $T$ and a $B$ (charge $\left.-\frac{1}{3}\right)$, and a $(3,1)$ model containing a $T, B$ and $X_{-\frac{4}{3}}\left(\right.$ charge $\left.-\frac{4}{3}\right) .^{3}$ None of these states have exotic-looking decays, and thus these models present the most challenging experimental signatures. Higher-dimension representations necessarily include states with charge not equal to $\frac{1}{3}, \frac{2}{3}$, or $\frac{4}{3}$, decaying via multiple $W$ bosons to like-sign leptons [21]. This is a more straightforward experimental signature, which is already being explored at the LHC [34, 42, 43] (see section 3.4).

\subsection{Singlet model}

Our first model, already described in [29] (where it is called ' $\mathrm{M} 1_{5}$ ') has the advantages of featuring custody of $Z \rightarrow b \bar{b}$ and full $t_{R}$ compositeness. The model has the following fields: $Q_{L}$, carrying the 5-d $(1,0)$ irrep of $\operatorname{Sp}(2)$ and containing the elementary $t_{L}$ and $b_{L} ; \Psi$, carrying the 1 -d $(1,1)^{-}$irrep of $\mathrm{SU}(2)^{2} \rtimes \mathbb{Z}_{2}$ and containing one vector-like top partner $T$;

\footnotetext{
${ }^{3}$ Note that the triplet charge assignments are incompatible with custody of $Z \rightarrow b \bar{b}$, which requires charges of $-\frac{1}{3}, \frac{2}{3}$, and $\frac{5}{3}$ for the $((1,3))$ irrep.
} 
$t_{R}$, also carrying the $(1,1)^{-}$irrep of $\mathrm{SU}(2)^{2} \rtimes \mathbb{Z}_{2}$ and containing the composite $t_{R}{ }^{4,5}$ and the coset representative, $U$, built out of the four real Higgs fields (the exact embeddings are given in [29]). The effective Lagrangian is

$$
\begin{aligned}
\mathcal{L}= & i \overline{Q_{L}} \not D Q_{L}+i \overline{t_{R}} \not D t_{R}+\bar{\Psi}(i \not D-M) \Psi \\
& -y f \overline{Q_{L}} U \Psi_{R}-y c_{2} f \overline{Q_{L}} U t_{R}+\text { h.c }+\ldots,
\end{aligned}
$$

parametrised by $\left\{f, M, y, c_{2}\right\}$, of which $c_{2}$ is fixed to obtain the correct top mass as follows. After EWSB, the charge $\frac{2}{3}$ states mix according to the matrix

$$
\mathcal{L} \supset-\left(\overline{t_{L}} \overline{T_{L}}\right)\left(\begin{array}{cc}
\frac{y c_{2} v}{\sqrt{2}} & \frac{y v}{\sqrt{2}} \\
0 & M
\end{array}\right)\left(\begin{array}{c}
t_{R} \\
T_{R}
\end{array}\right) .
$$

where $v=246 \mathrm{GeV}$. The mass matrix may be diagonalised by a singular value decomposition, where the physical masses are given by the singular values. We require the smaller singular value to be the top mass, which implicitly fixes $c_{2}$ as a function of $M$ and $y$; the larger value - now a function of $M$ and $y$ alone - gives the mass of the top partner.

The top partners are pair produced by QCD interactions, and hence the pair production cross-section at leading order depends only on the top partner mass. They may also be singly produced, with LO cross-section proportional to $y^{2}$. It is found that the top partner production cross-section does not strongly depend on the value of $f$ (which we set to $500 \mathrm{GeV}$ ). We thus consider the free parameters of the model to be $m_{T}$ and $y$.

There are 3 possible decay modes for the $T: W b, Z t$ or $H t$; their branching fractions are roughly $2: 1: 1{ }^{6}$ The final state of singly or pair-produced $T$ of mass of a few hundred $\mathrm{GeV}$ thus invariably contains several high $p_{T}$ objects, including several $b$-jets, as well as a reasonable branching fraction to leptons.

\subsection{Doublet model}

The next model contains a top partner carrying the $(1,2)$ irrep of $\mathrm{SU}(2)^{2}$. Unlike the singlet model, this cannot feature custody of $Z \rightarrow b \bar{b}$; nor can the $t_{R}$ be fully composite. Neither can be considered a sine qua non, given the tunings already required in the electroweak $S$ parameter and flavour sector and moreover this model provides a sufficiently different experimental signature to merit consideration, especially if it can be covered by the same experimental search as the singlet model. Summarising appendix B, this requires elementary fields $Q_{L}, t_{R}$ and $b_{R}$, each carrying the $\left(\frac{1}{2}, \frac{1}{2}\right)$ rep of $\operatorname{Sp}(2)$ and containing respectively the $t_{L}$ and $b_{L}$, the $t_{R}$, and the $b_{R}$. The top partner field, $\Psi$, contains a $T$ and a $B$. The Lagrangian is

$$
\begin{aligned}
\mathcal{L}= & i \overline{Q_{L}} \not D Q_{L}+i \overline{t_{R}} \not D t_{R}+i \overline{b_{R}} \not D b_{R}+\bar{\Psi}(i \not D-\phi-M) \Psi \\
& -y f \overline{Q_{L}} U \Psi_{R}-y_{R}^{t} f \overline{t_{R}} U \Psi_{L}-y_{R}^{b} f \overline{b_{R}} U \Psi_{L}+\text { h.c }+\ldots
\end{aligned}
$$

\footnotetext{
${ }^{4}$ Desiring to avoid overloading the reader's RAM, and at the risk of incurring kernel panic, we use $t_{R}$ to represent both the Weyl fermion and the vector that gives its embedding in the 5 rep of $\operatorname{Sp}(2)$ : $t_{R}=\left(0,0,0,0, t_{R}\right)^{T}$.

${ }^{5}$ As in [29], the $b_{R}$ is absent from our discussion.

${ }^{6}$ This is understood [29] by considering the interaction $y f \overline{Q_{L}} U \Psi_{R}$ of Lagrangian (3.1). In the limit of a heavy $\Psi$, the Goldstone boson equivalence theorem predicts equal decays to $W^{1}, W^{2}, Z$ and $h$.
} 
The model has parameters $\left\{f, M, y, y_{R}^{t}, y_{R}^{b}\right\}$; the latter two of these are fixed by the top and bottom masses after singular value decomposition of the mass matrices.

Again, the phenomenology does not depend strongly on the value of $f$, which is set to be $500 \mathrm{GeV}$, so the free parameters may again be taken to be $M$ and $y$. For large $y$, the masses of the $T$ and $B$ are very similar in value, whilst the $T$ is significantly heavier at small $y$. The $T$ decay channels are as in the singlet model, plus possibly a small branching fraction for $T \rightarrow B W$ : the branching fraction to $T \rightarrow h t$ dominates $(\sim 50-80 \%$ in the parameter space considered). The $B$ has three decay modes: $B \rightarrow W t, B \rightarrow Z b, B \rightarrow h b$. The decay via a $W$ boson is the most likely, with a branching fraction of $\sim 50-80 \%$; the other two decays are equally likely. The phenomenology is thus more varied than for the singlet model, but the most distinctive features are still present: large $b$-jet multiplicity and some likelihood of leptonic $W$-boson decays at high mass.

\subsection{Triplet models}

As described above, there are also triplet models with top partners whose charges only equal $\frac{1}{3}, \frac{2}{3}$, or $\frac{4}{3}$, viz. $(1,3)$ or $(3,1)$ irreps. The novel feature of these models is that their lightest top partner, in the absence of similarly charged states to mix with, has charge $\pm \frac{4}{3}$, and will overwhelmingly decay to $b$ quarks via 'wrong-sign' $W$-bosons. Excepting the unmeasured charge of the $\mathrm{b}$ quark, this is the same $W b$ signature which is targeted in the case of the singlet model. Compared to the singlet case, however, the signal is enhanced by virtue of $\operatorname{Br}(\rightarrow W b)=1$ and by the additional contribution of the triplet's charge $\frac{2}{3}$ state. The same search strategy will thus give limits that are at least as good as the singlet model for corresponding masses, and we do not consider them further here.

\subsection{Larger representation models}

As all less minimal models contain states with charges not equal to $\frac{1}{3}, \frac{2}{3}$, or $\frac{4}{3}$ (such as $\frac{5}{3}, \ldots$ ), they present a more straightforward experimental signature, in the form of same-sign leptons, and searches are already being performed by the ATLAS and CMS Collaborations [42, 43].

Not only are these states easily identifiable, but they are also the lightest, as we now prove. In our effective field theory approach, we integrate out all but the top partner $\Psi$, i.e. the lightest fermionic resonance of the strong sector. ${ }^{7}$ For (a) a partially composite and (b) a fully composite $t_{R}$, for example, the mass terms in the Lagrangian are:

(a) $\mathcal{L} \supset-M \overline{\Psi_{L}} \Psi_{R}-y_{1} f \overline{Q_{L}} U \Psi_{R}-y_{2} f \overline{Q_{L}} U t_{R}+$ h.c.,

(b) $\mathcal{L} \supset-M \overline{\Psi_{L}} \Psi_{R}-y_{1} f \overline{Q_{L}} U \Psi_{R}-y_{2} f \overline{t_{R}} U \Psi_{L}+$ h.c..

\footnotetext{
${ }^{7}$ It is thus an assumption of our EFT analysis that all other states are significantly heavier. If there are two or more top partner multiplets with comparable mass terms $M$, some non-exotically charged states may receive negative $\mathrm{O}\left(\frac{y^{2} v^{2}}{M}\right)$ contributions to their mass on mixing, where $y$ is a generic Yukawa coupling and $v$ the electroweak scale. For such models the exotic states remain among the lightest new states; at worst they are slightly heavier than other new, but harder to see, resonances.
} 
The mass matrices for the charge $\frac{2}{3}$ states are thus

(a) $\left(\begin{array}{lllll}t_{L} & T_{L}^{1} & T_{L}^{2} & \ldots & T_{L}^{n}\end{array}\right)\left(\begin{array}{ccccc}a & a_{1} & a_{2} & \ldots & a_{n} \\ 0 & M & 0 & & \\ 0 & 0 & M & & \\ \vdots & & & \ddots & \\ 0 & & & & M\end{array}\right)\left(\begin{array}{c}t_{R} \\ T_{R}^{1} \\ T_{R}^{2} \\ \vdots \\ T_{R}^{n}\end{array}\right)$,

(b) $\left(\begin{array}{lllll}t_{L} & T_{L}^{1} & T_{L}^{2} & \ldots & T_{L}^{n}\end{array}\right)\left(\begin{array}{ccccc}0 & a_{1} & a_{2} & \ldots & a_{n} \\ b_{1} & M & 0 & & \\ b_{2} & 0 & M & & \\ \vdots & & & \ddots & \\ b_{n} & & & & \\ M\end{array}\right)\left(\begin{array}{c}t_{R} \\ T_{R}^{1} \\ T_{R}^{2} \\ \vdots \\ T_{R}^{n}\end{array}\right)$,

whose singular values under singular value decomposition give the fermion masses. $a_{i} \sim$ $\mathrm{O}\left(y_{1} v\right)$ and $b_{i} \sim \mathrm{O}\left(y_{2} v\right)$ with $v$ the SM Higgs v.e.v., and assuming $a_{i}, b_{i} \ll M$ then in both cases (a) and (b) the resulting spectrum of masses is $M$ (with degeneracy $n-1$ ) together with 1 value $\leq M$ (which we identify with $m_{t}$ ) and one value $\geq M$. The same is true for the charge $-\frac{1}{3}$ states. This can be seen explicitly for case (b), for example, as follows. By suitable rotations of the left and right handed composite states the mass matrix (b) may be put in the form:

$$
\left(\begin{array}{ccccc}
0 & a & 0 & \ldots & 0 \\
b & M & 0 & & \\
0 & 0 & M & & \\
\vdots & & & \ddots & \\
0 & & & & M
\end{array}\right)
$$

which has $n-1$ singular values equal to $M$, and the other two are the singular values of $\left(\begin{array}{ll}0 & a \\ b & M\end{array}\right)$, namely

$$
\sqrt{\frac{1}{2}\left(M^{2}+a^{2}+b^{2}\right) \pm \frac{1}{2} \sqrt{\left(M^{2}+a^{2}+b^{2}\right)^{2}-4 a^{2} b^{2}}} \approx \begin{cases}\sqrt{M^{2}+a^{2}+b^{2}}, & \text { top partner } \\ \frac{a b}{M}, & \text { top. }\end{cases}
$$

These solutions are, respectively, $>M$ and $<M$. Note also that, in the case where the model contains a charge $-\frac{1}{3} B$ as well as a charge $\frac{2}{3} T$ top partner, the values $a_{i}$ and $M$ are common to both charge $-\frac{1}{3}$ and $\frac{2}{3}$ mass matrices. The $b_{i}$ are adjusted to produce a bottom mass smaller than that of the top, and in doing so produce a $B$ lighter than the $T$. A similar proof holds for case (a).

In contrast to the charge $-\frac{1}{3}$ and $\frac{2}{3}$ composite states, the other states have nothing to mix with, and have mass $M$, which is the lightest top partner mass. 


\section{Current experimental limits on the singlet and doublet models}

Current experimental limits can be divided into 2 categories: those from dedicated searches for $T$ and $B$, and those from other analyses searching for other models, but considering similar final-state signatures. Particularly relevant are SUSY searches requiring high effective mass, high $b$-jet multiplicity, and leptons. We find that the limits from the dedicated searches are generally the strongest, as might be expected, but that at large coupling $y$, the SUSY searches become competitive. We now describe the searches and our reinterpretation of their limits. The results are shown in section $6 .^{8}$

\subsection{SUSY searches}

As most SUSY searches are "cut-and-count", they can be readily reinterpreted in the context of composite Higgs models through the raw event count in the signal regions. For each model, a grid of signal points is simulated using FeynRules [44] and MADGRAPH 5 v1.5.12 [45]. The hadronic shower was simulated using PyтнIA 6 [46], and the response of the ATLAS detector was simulated using Delphes 3 [47]. The same cuts as for the searches are applied, and limits are then set by comparing the observed and expected number of events from the published search with the signal expectation, using the $C L_{s}$ prescription [48].

Of all the public searches for SUSY by the ATLAS and CMS Collaborations that we tested, 3 were found to be reasonably sensitive. They are the ATLAS " $0 \ell+2 b$-jets + $E_{T}^{\text {miss" }}[49]$, the ATLAS " $1 \ell+2-6$ jets" [50] and the CMS " $1 \ell+6-7$ jets $+b$-jets" [51] searches.

\subsection{Dedicated searches}

Both the ATLAS and CMS Collaboration have performed dedicated searches for $T$ and $B$ top partners [52-54]. These searches only target the pair-production of the new states in one or two very specific decay channels, and set limits on their masses in terms of their branching fractions. These limits can then be used directly to set limits on the models considered here. These searches make use of advanced techniques that are not easily replicable, such as BDTs, jet substructure and hadronic $W$-tagging; we believe these results can be reproduced more simply and effectively using the search in section 6 .

\section{$5 \quad$ New analysis design}

Expanding upon some of the search strategies already implemented by the LHC experiments, we show that it is possible to set stringent limits on both the singlet and the doublet model with one rather straightforward and inclusive search. We estimate limits on the current models from this search for both the current LHC dataset $\left(20.3 \mathrm{fb}^{-1}\right.$ at $\left.\sqrt{s}=8 \mathrm{TeV}\right)$, as well as after a year of running at higher energies $\left(30 \mathrm{fb}^{-1}\right.$ at $\left.\sqrt{s}=14 \mathrm{TeV}\right)$. All signal samples are simulated using MAdGraph 5 v1.5.12, showered with Pythia 6, and the

\footnotetext{
${ }^{8}$ Considering just pair production, model-independent limits on top partners from both SUSY and dedicated searches can be found in [37] (in terms of their masses and branching fractions).
} 
ATLAS detector response is simulated using Delphes 3. Jets are reconstructed using the anti- $k_{T}$ algorithm with $R=0.5$.

For the $\sqrt{s}=8 \mathrm{TeV}$ analysis, events are required to contain exactly 1 lepton, at least $1 b$-jet and at least 5 jets in total. Furthermore, events must have $E_{T}^{\text {miss }}>50 \mathrm{GeV}$, the leading jet must have $p_{T}>400 \mathrm{GeV}$ and $m_{\mathrm{Eff}}>1300 \mathrm{GeV} .{ }^{9}$ The $\sqrt{s}=14 \mathrm{TeV}$ analysis differs from this only in that the jet $p_{T}$ cut is raised to $600 \mathrm{GeV}$, and the $m_{\text {Eff }}$ cut to $1500 \mathrm{GeV}$. In addition to these cuts, both analyses use a "top veto", requiring that the minimum reconstructed mass of the decay $X \rightarrow W b \rightarrow \ell \nu b, m_{\ell \nu b}$, be above $200 \mathrm{GeV}$. The analysis cuts are summarised in table 2 .

The top veto, being the most unusual feature of the analysis, deserves some further discussion. The invariant mass of the decay is reconstructed by first solving for the $z$ component of the missing transverse momentum, using the assumption that the lepton and missing energy come from the leptonic decay of a $W$ boson (of known mass $m_{W}$ ). To do so requires us to solve a quadratic equation, and thus there is a twofold ambiguity in the resulting $W$-boson 4 -momentum. These two 4-momenta are then added in turn to each possible $b$-jet 4 -momentum in the event. The 4 -momentum with the minimum invariant mass, $m_{\ell \nu b}$, that results is selected. For illustration, the distribution of $m_{\ell \nu b}$ is shown in figure 1. The selection cuts are somewhat loosened with respect to the signal selection to increase statistics: 3 instead of 5 jets, leading jet satisfying $p_{T}>300 \mathrm{GeV}$ instead of $p_{T}>400 \mathrm{GeV}, m_{\mathrm{Eff}}>1000 \mathrm{GeV}$ instead of $1300 \mathrm{GeV}$, and no $m_{\ell \nu b}$ cut.

Requiring that this mass lies somewhat above the mass of the top quark will give a significant rejection of the SM semi-leptonic $t \bar{t}$ background. Assuming perfect detector reconstruction, roughly half of the background can be rejected in this way (since there are two $b \mathrm{~s}$ in the event). The signal suppression is typically rather less. This is easily seen by considering the possible signal final states in turn. The worst cases are $B \rightarrow b Z$ and $B \rightarrow b h$, which contain 0 or 2 leptons, and are vetoed (we require exactly 1 lepton to reduce the SM $Z+$ jets background). But these tend to have small branching ratio. For $T \rightarrow t Z$, there can be exactly 1 lepton from the top, but then the probability to reconstruct a top is comparable to that for the background. More promising is pair production of $B \rightarrow W t$, where there are twice as many $W \mathrm{~s}$ in the event, compared to the $t \bar{t}$ background, and so the probability that the lepton came from a $t$ quark decay is reduced by a factor of one-half. For events involving $T \rightarrow t H$, there are extra $b$ jets coming from the Higgs decay that make it combinatorially unlikely to reconstruct a $t$. For other decays, $m_{\ell \nu b}$ will typically be much greater than $m_{t}$.

Thus we already typically gain a factor of a few in the ratio of signal to background from the top veto in a given production and decay mode. An even greater gain comes from the inclusivity of the search that it allows. Whereas existing searches tune the cuts to focus on a particular production mode (usually pair production) of a particular state ( $T$ or $B$ ) with a particular decay mode, our proposed search is sensitive to almost all of them. Given that the states are generically not too dissimilar in mass, and given that the branching ratios to different final states are typically comparable, this leads to rather enhanced sensitivity, which we expect to see translated into higher limits.

\footnotetext{
${ }^{9}$ The scalar sum of the transverse momenta of all selected jets and leptons as well as the $E_{T}^{\text {miss }}$.
} 


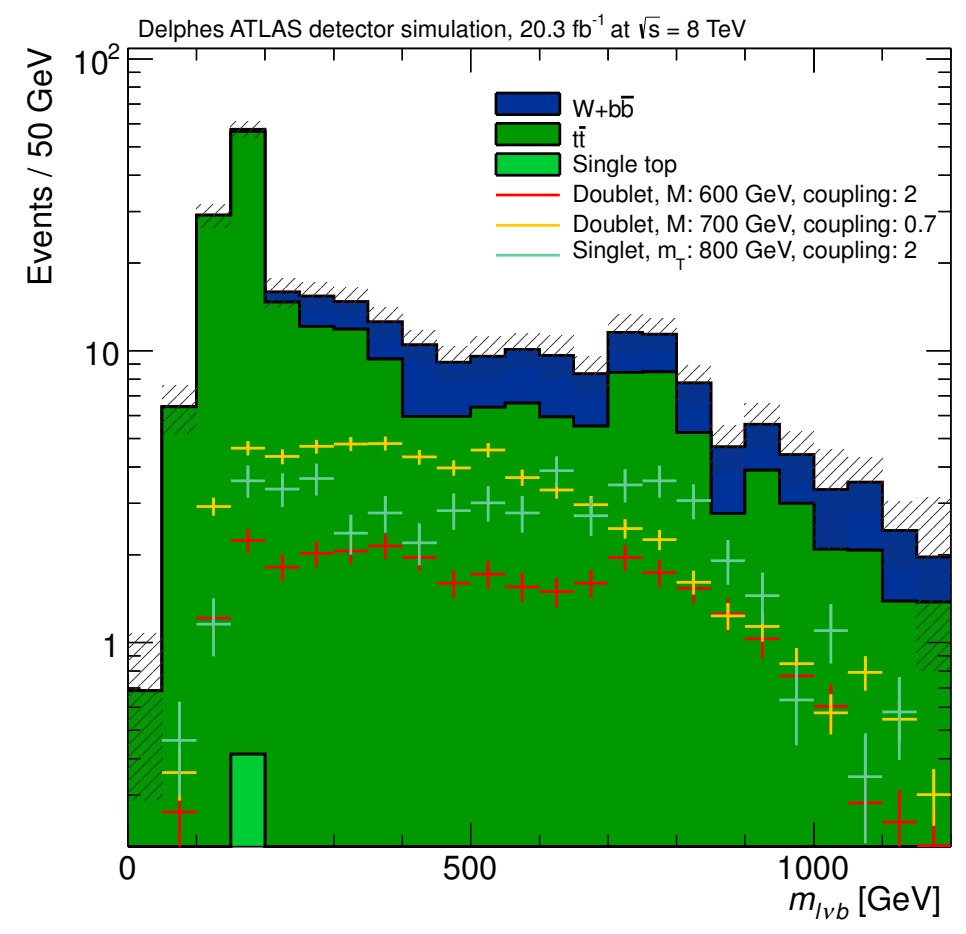

Figure 1. Reconstructed mass, $m_{\ell \nu b}$, for $20.3 \mathrm{fb}^{-1}$ at $\sqrt{s}=8 \mathrm{TeV}$, with several superimposed signal distributions for both the singlet and doublet models. To increase statistics, the event selection for this plot is somewhat loosened from the signal selection (for details, see the text).

\begin{tabular}{|c|c|}
\hline \multicolumn{2}{|c|}{ Object Selection } \\
\hline Object & Requirement \\
\hline Electrons & $p_{T}>20 \mathrm{GeV},|\eta|<2.47$ \\
\hline Muons & $p_{T}>20 \mathrm{GeV},|\eta|<2.4$ \\
\hline Jets & $\begin{array}{c}\text { anti- } k_{t} \text { jets, } R=0.5 \\
p_{T}>40 \mathrm{GeV},|\eta|<2.8\end{array}$ \\
\hline Isolation & $\begin{array}{l}\text { Objects need to be isolated from } \\
\text { each-other by } \Delta R>0.5\end{array}$ \\
\hline \multicolumn{2}{|c|}{ Event Selection } \\
\hline \multicolumn{2}{|c|}{ exactly one lepton } \\
\hline \multicolumn{2}{|c|}{ number of $b$-jets $\geq 1$} \\
\hline \multicolumn{2}{|c|}{$E_{T}^{\text {miss }}>50 \mathrm{GeV}$} \\
\hline \multicolumn{2}{|c|}{ number of jets $\geq 5$} \\
\hline \multicolumn{2}{|c|}{$m_{\ell \nu b}>200 \mathrm{GeV}$} \\
\hline$\sqrt{s}=8 \mathrm{TeV}$ & $\sqrt{s}=14 \mathrm{TeV}$ \\
\hline leading jet $p_{T}>400 \mathrm{GeV}$ & leading jet $p_{T}>600 \mathrm{GeV}$ \\
\hline$m_{\mathrm{Eff}}>1300 \mathrm{GeV}$ & $m_{\mathrm{Eff}}>1500 \mathrm{GeV}$ \\
\hline
\end{tabular}

Table 2. Analysis selection for the $8 \mathrm{TeV}$ analysis. The events are passed through the DeLPhes ATLAS detector simulation. 
The main backgrounds to this analysis are $t \bar{t}$ and $W+b \bar{b}$ (roughly equal proportion), with a small contribution from single top processes, all of which are simulated using the same chain as the signal samples. Contributions from $Z+$ jets processes are vetoed by requiring exactly one lepton, and di-boson contribution is found to be negligible, compatible with the findings of [53], which has a similar selection.

\section{Limits on the singlet and doublet models}

Limits are set using a $C L_{s}$ test, and assuming that the observed data would be the same as the expected background. Statistical uncertainties are assessed on both the background expectation and the signal prediction. To estimate the systematic uncertainties on the background expectation, e.g. from the choice of generator or from experimental uncertainties, we use the uncertainties on the backgrounds given in [53], where a similar selection was used. Thus, we assign a $60 \%$ systematic uncertainty on the $t \bar{t}$ background, and a $42 \%$ uncertainty on the other backgrounds. The cuts for the analysis were optimised, though the chosen cuts on some variables such as $m_{\mathrm{Eff}}$ were not as stringent as they could have been, as the background estimation on the tails of the distributions becomes unreliable. In a real experimental search, the background estimation including fake leptons would be done more carefully and with more computational resources, and thus a more robust estimate of the backgrounds would be possible. Thus, the results presented in this section should be regarded as a rough estimate of the actual limits that such an analysis would yield at at $\sqrt{s}=8 \mathrm{TeV}$ and $\sqrt{s}=14 \mathrm{TeV}$. The exclusions obtained with the new analysis for the singlet and doublet models, both at $\sqrt{s}=8 \mathrm{TeV}$ and $\sqrt{s}=14 \mathrm{TeV}$, are shown in figures 2 and 3 .

As expected, the use of the top veto and the gain in inclusivity of the search suggested here yield significantly better limits than existing searches that target only one decay mode, with dramatically decreased acceptance. This is especially true for the limits on the doublet model obtained using dedicated searches.

We note from figures 2 and 3 that the limits on the top partner masses increase with $y$, due to the contribution of single production. Though hard to quantify, the single production mode becomes more relevant as we seek to exclude masses closer to the CoM energy of the LHC; at the moment, it can provide a model-dependent $\mathrm{O}(100 \mathrm{GeV})$ increase in the exclusion limit. Our inclusive search is sensitive to its effects; however, we require large $m_{\mathrm{Eff}}$ of the events, and for decays to tops, we rely primarily on pair production to provide many high $p_{T}$ objects, so that the top is incorrectly reconstructed and the top veto is thus avoided.

\section{Discussion}

We have presented a classification of the lightest fermionic resonances, or top partners, in models in which the Higgs boson is a composite resonance of strongly-coupled dynamics. The top partners are expected to be reasonably light (and within the reach of the LHC), in order to reproduce the measured Higgs boson mass. We find that there are restrictions of the possible charges of top partners if we insist either on a custodial symmetry to protect 


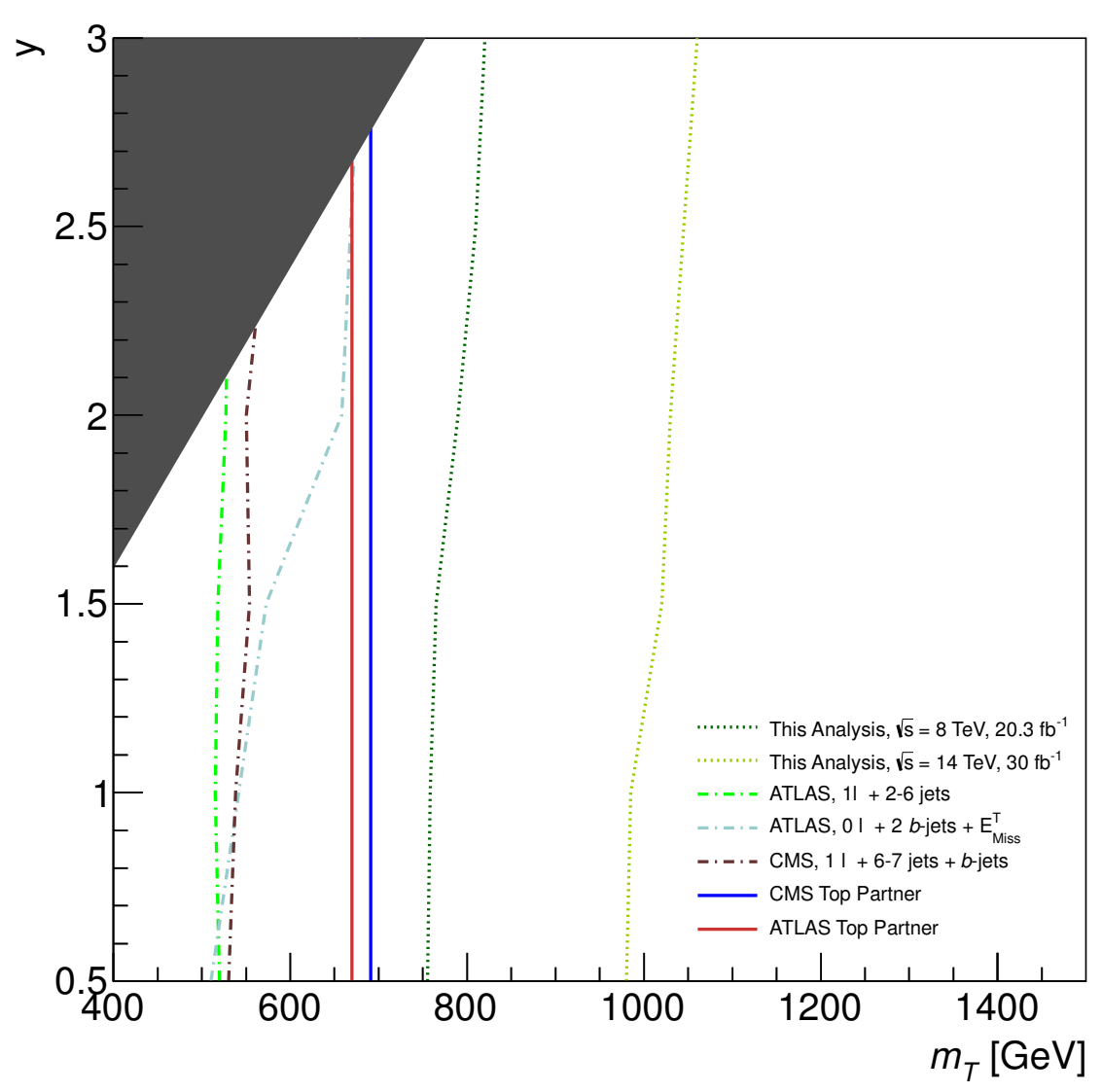

Figure 2. Exclusion limits on the singlet model obtained from published analyses from the experiments (solid), from reinterpretations of analyses not explicitly interpreted in the context of top partners (dot-dashed), and projected exclusions with the analysis proposed here at $\sqrt{s}=8 \mathrm{TeV}$ and $\sqrt{s}=14 \mathrm{TeV}$ (dotted). In the dark grey region, the constraint from the known top quark mass cannot be satisfied.

the rate for $Z \rightarrow b \bar{b}$, or that the $t_{R}$ be wholly composite. In the first case, the top partner must transform in either the $(2 l+1,2 l+1)^{ \pm}$or the $((2 l+1,2 l+3))$ irrep of the custodial symmetry group $\mathrm{SU}(2)^{2} \rtimes \mathbb{Z}_{2}$; in the second case, the top partner is restricted to the $(2 l+1,2 l+1)$ irrep of $\mathrm{SU}(2)^{2}$.

Using this classification, we have explored possible experimental searches for top partners at the LHC. While top partners with electric charges not equal to $\pm \frac{1}{3}, \pm \frac{2}{3}$, or $\pm \frac{4}{3}$ should be relatively easy to find via excesses in events with like-sign dileptons, other top partners, decaying to $t$ or $b$ quarks and $W, H$ or $Z$ bosons with comparable branching fractions, may prove more difficult. For these, we find that a single search that is based on a top quark veto, but that it is otherwise reasonably inclusive, gives good sensitivity throughout the space of models and parameters. Thus, it should be relatively straightforward to either discover or exclude the composite Higgs hypothesis at the LHC, by means of two generic searches. 


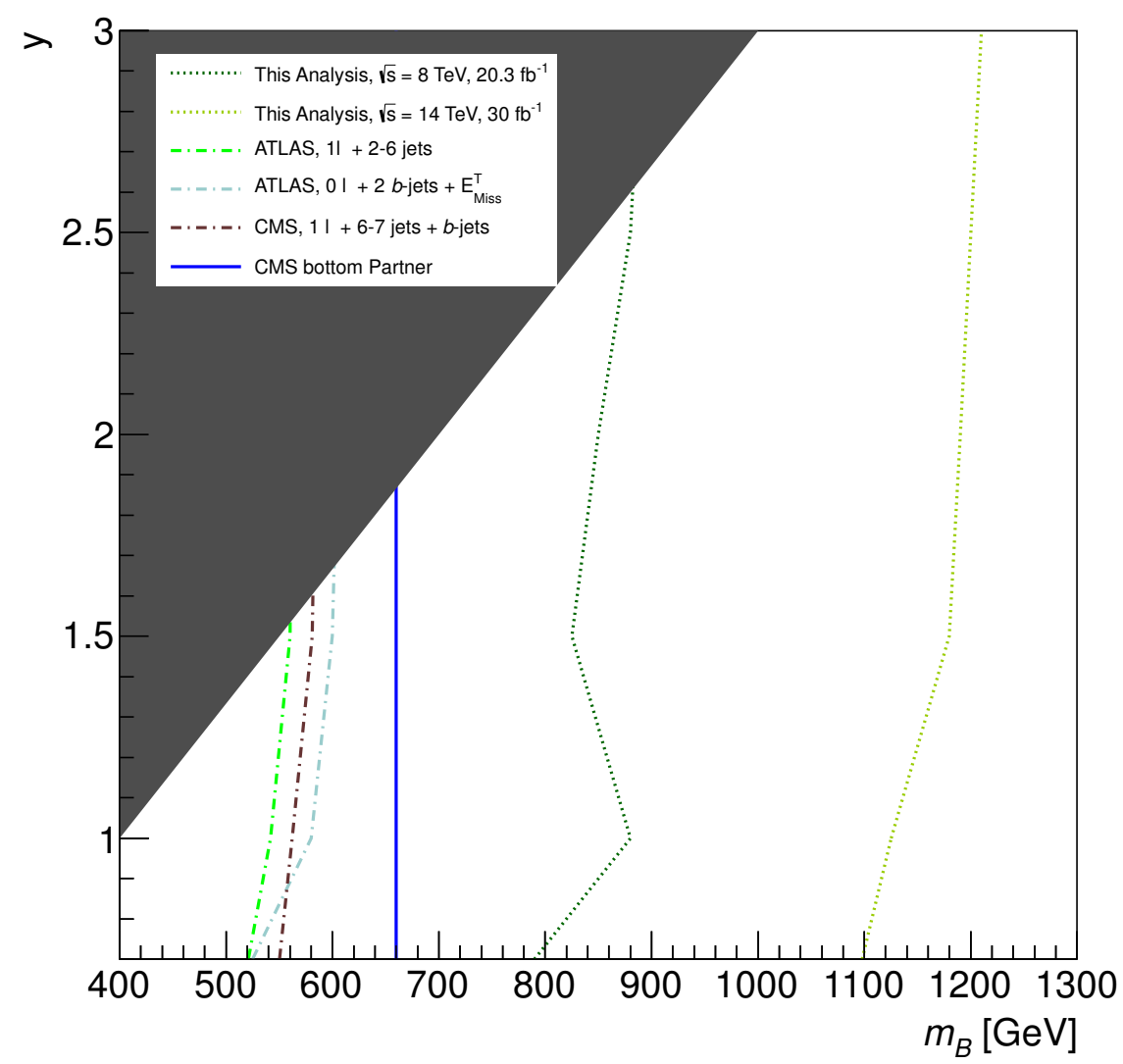

Figure 3. Exclusion limits on the doublet model obtained from published analyses from the experiments (solid), from reinterpretations of analyses not explicitly interpreted in the context of top partners (dot-dashed), and projected exclusions with the analysis proposed here at $\sqrt{s}=8 \mathrm{TeV}$ and $\sqrt{s}=14 \mathrm{TeV}$ (dotted). In the dark grey region, the constraint from the known top and bottom quark masses cannot be satisfied.

\section{Acknowledgments}

BG acknowledges the support of the Science and Technology Facilities Council, the Institute for Particle Physics Phenomenology, and King's College, Cambridge and thanks R. Contino and R. Rattazzi for discussions. DS acknowledges the support of the Science and Technology Facilities Council, as well as Emmanuel College, Cambridge, and thanks O.Matsedonskyi for FeynRules help. TM thanks C. Lester for discussions on mass variables.

\section{A Group and representation theory of top partners}

In this appendix, we discuss the necessary group and representation theory for the classification of top partners in a composite Higgs model based on the coset $G / H$ given in section 2 . We begin by considering $H$ to be locally isomorphic to $\mathrm{SU}(2)^{2}$ and to be both connected and simply-connected, i.e. $H \simeq \mathrm{SU}(2)^{2}$. If $H$ contains $\mathrm{SU}(2)^{2}$ as a proper sub- 
group, we can make similar arguments, except that we should replace $H$ by its $\mathrm{SU}(2)^{2}$ subgroup and then pick out from the representations thus obtained only those that can be lifted to reps of $H$. Similarly, if $H$ is not simply-connected, then $H$ (or a subgroup thereof) can be obtained by taking the quotient of $\mathrm{SU}(2)^{2}$ with a non-trivial subgroup of its centre, $\mathbb{Z}_{2}^{2}$. These quotients are isomorphic to $\mathrm{SU}(2) \times \mathrm{SO}(3), \mathrm{SO}(3)^{2}$, and $\mathrm{SO}(4)$ (which is obtained by taking the quotient with the diagonal $\mathbb{Z}_{2}$ subgroup of $\left.\mathbb{Z}_{2}^{2}\right) .{ }^{10}$ The only change in the results obtained below is that the possible irreps for the top partner are limited to those irreps of the covering group $\mathrm{SU}(2)^{2}$ that restrict to the identity on the subgroup used to form the quotient, namely those $(2 l+1,2 r+1)$ with even values of $2 r, 2 l$ and $2 r$, and $2(l+r)$, respectively.

If we wish to protect the rate for $Z \rightarrow b \bar{b}$, then it is useful to also consider the case where $H$ is a (disconnected) semi-direct product of $\mathrm{SU}(2)^{2}$ with $\mathbb{Z}_{2}$. Recall that given two groups, $N$ and $K$, and a homomorphism $\phi: K \rightarrow \operatorname{Aut}(N)$, the semi-direct product group $N \rtimes K$ is the set $N \times K$ together with the multiplication operation defined by $\left(n_{1}, k_{1}\right) \cdot\left(n_{2}, k_{2}\right) \equiv\left(n_{1} \phi_{k_{1}}\left(n_{2}\right), k_{1} k_{2}\right)$. Here, we let $N=\mathrm{SU}(2)^{2}$ and $K=\mathbb{Z}_{2}$, with $\phi$ mapping $\mathbb{Z}_{2}$ to the (outer) automorphisms of $N$ obtained by permuting the two SU(2)s. ${ }^{11}$

The irreps of SU(2) $\rtimes \mathbb{Z}_{2}$ can be obtained mechanically by inducing them from the irreps of the subgroups $\mathrm{SU}(2)^{2}$ and $\mathbb{Z}_{2}$, but it is easy enough to guess the irreps directly and then prove that they are all irreps. The result is that there are three classes of irreps. Two of these classes, which we denote by $(2 l+1,2 l+1)^{ \pm}$, are equivalent to

$$
\begin{aligned}
& D^{ \pm}\left(g_{L}, g_{R},+e\right)=D_{\alpha \beta}^{l}\left(g_{L}\right) D_{\dot{\alpha} \dot{\beta}}^{l}\left(g_{R}\right) \\
& D^{ \pm}\left(g_{L}, g_{R},-e\right)= \pm D_{\alpha \dot{\beta}}^{l}\left(g_{L}\right) D_{\dot{\alpha} \beta}^{l}\left(g_{R}\right)
\end{aligned}
$$

where $D^{l}(g)$ is any matrix representing the $\mathrm{SU}(2)$ element $g$ in the irrep $2 l+1$. When restricted to $\mathrm{SU}(2)^{2}$, both of these irreps reduce to the direct product irrep, but they are inequivalent as irreps of $\mathrm{SU}(2)^{2} \rtimes \mathbb{Z}_{2}$. The third class of irreps, which we denote by $((2 l+1,2 r+1))$ with $l \neq r$, are given by

$$
\begin{aligned}
& D\left(g_{L}, g_{R},+e\right)=\left(\begin{array}{cc}
D^{l}\left(g_{L}\right) \otimes D^{r}\left(g_{R}\right) & 0 \\
0 & D^{l}\left(g_{R}\right) \otimes D^{r}\left(g_{L}\right)
\end{array}\right) \\
& D\left(g_{L}, g_{R},-e\right)=\left(\begin{array}{cc}
0 & D^{l}\left(g_{L}\right) \otimes D^{r}\left(g_{R}\right) \\
D^{l}\left(g_{R}\right) \otimes D^{r}\left(g_{L}\right) & 0
\end{array}\right) .
\end{aligned}
$$

Their restriction to $\mathrm{SU}(2)^{2}$ is a direct sum, $(2 l+1,2 r+1) \oplus(2 r+1,2 l+1)$.

\footnotetext{
${ }^{10}$ These results are easily established by means of the following explicit homomorphisms: (i) Consider $(x, y, z) \in \mathbb{R}^{3}$ and let $M \equiv\left(\begin{array}{cc}z & x+i y \\ x-i y & z\end{array}\right)$. Then $U \in \mathrm{SU}(2)$ acting as $M \mapsto U M U^{*}$ effects an orthogonal transformation of determinant +1 on $\mathbb{R}^{3}$ with kernel $U \in\{I,-I\}$; (ii) Consider $(x, y, z, w) \in \mathbb{R}^{4}$ and let $M \equiv\left(\begin{array}{cc}z+i w & x+i y \\ -x+i y & z-i w\end{array}\right)$. Then $(U, V) \in \mathrm{SU}(2) \times \mathrm{SU}(2)$ acting as $M \mapsto V M U^{-1} \operatorname{preserves} \operatorname{det} M=$ $x^{2}+y^{2}+z^{2}+w^{2}$ and so is an orthogonal transformation on $\mathbb{R}^{4}$ with kernel $(U, V) \in\{(I, I),(-I,-I)\}$. Direct computation establishes that the map has determinant +1 .

${ }^{11} \mathrm{In}[8]$, this group is written as $\mathrm{SU}(2) \otimes \mathrm{SU}(2) \otimes P_{L R}$.
} 
We prove that (A.1)-(A.2) are all irreps by standard methods in the representation theory of compact Lie groups [55], showing that the characters of the irreps form a complete, orthonormal set of functions on the conjugacy classes of the group.

We warm up with $\mathrm{SU}(2)$, where every element is conjugate (denoted by $\sim$ ) to an element of the form $\operatorname{diag}\left(e^{i t}, e^{-i t}\right)$. Now $t \sim t+2 \pi$ and moreover $t \sim-t$, as can be shown by conjugating with $\left(\begin{array}{cc}0 & 1 \\ -1 & 0\end{array}\right) \in \mathrm{SU}(2)$. So the class functions are even, periodic functions on $\mathbb{R}$. Now, the usual irrep with multiplicity $2 j+1$ has class representative $\operatorname{diag}\left(e^{2 i j t}, e^{2 i(j-1) t}, \ldots, e^{-2 i j t}\right)$ and character $\chi^{j}(t)=\frac{\sin (2 j+1) t}{\sin t}=\cos 2 j t+\chi^{j-\frac{1}{2}} \cos t$, so it is clear that by taking linear combinations with different $j$, we may obtain the complete set of even, periodic functions $1, \cos t, \cos 2 t, \ldots$ exactly once. Finally, the normalized groupinvariant measure on $\mathrm{SU}(2)$ is the same as the one obtained from the round metric on $S^{3}$, viz. $\frac{1}{2 \pi^{2}} \int_{0}^{\pi} \sin ^{2} t d t \int_{0}^{\pi} \sin \theta d \theta \int_{0}^{2 \pi} d \phi$, which reduces to $\frac{2}{\pi} \int_{0}^{\pi} d t \sin ^{2} t$ on class functions. Since the characters of the above reps of $\mathrm{SU}(2)$ satisfy

$$
\frac{2}{\pi} \int_{0}^{\pi} d t \sin ^{2} t \chi^{j *}(t) \chi^{k}(t)=\delta^{j k}
$$

they are orthonormal and so they are indeed a complete set of irreps.

For $\mathrm{SU}(2)^{2}$, the classes may be represented by $\left(\operatorname{diag}\left(e^{i t_{L}}, e^{-i t_{L}}\right), \operatorname{diag}\left(e^{i t_{R}}, e^{-i t_{R}}\right)\right)$ and the character of rep $(2 l+1,2 r+1)$ is then the product of the corresponding $\mathrm{SU}(2)$ characters and the measure is the product of the two $\mathrm{SU}(2)$ measures. It is then immediate that these are inequivalent irreps and that they are all of the irreps.

For $\mathrm{SU}(2)^{2} \rtimes \mathbb{Z}_{2},\left(g_{L}, g_{R}, e\right) \sim\left(g_{R}, g_{L}, e\right)$ and so the classes in the component connected to the identity may be represented by $\left(\operatorname{diag}\left(e^{i t_{L}}, e^{-i t_{L}}\right), \operatorname{diag}\left(e^{i t_{R}}, e^{-i t_{R}}\right), e\right)$, where we restrict class functions to be even, periodic, and symmetric under the interchange $t_{L} \leftrightarrow t_{R}$. In the component that is not connected to the identity, we find that conjugating with $\left(h_{L}, h_{R}, e\right)$ and $\left(h_{L}, h_{R},-e\right)$ yields $\left(g_{L}, g_{R},-e\right) \sim\left(h_{L}^{-1} g_{L} h_{R}, h_{R}^{-1} g_{R} h_{L},-e\right)$ and $\left(g_{L}, g_{R},-e\right) \sim\left(h_{R}^{-1} g_{R} h_{L}, h_{L}^{-1} g_{L} h_{R},-e\right)$. The second of these conjugations with $h_{L}=g_{L}$ and $h_{R}=e$ implies that $\left(g_{L}, g_{R},-e\right) \sim(k, e,-e)$, where $k=g_{R} g_{L}$, and the first conjugation with $h_{L}=h_{R}=h$ then implies that $(k, e,-e) \sim\left(h^{-1} k h, e,-e\right)$. The upshot is that classes in the component disconnected from the identity can be parameterised similarly to those of $\mathrm{SU}(2)$, viz. as $\left(\operatorname{diag}\left(e^{i t}, e^{-i t}\right), e,-e\right)$, with class functions that are even and periodic in $t$.

The characters of the $(2 l+1,2 l+1)^{ \pm}$reps are given by

$$
\begin{aligned}
\chi^{ \pm}(+e) & =\frac{\sin (2 l+1) t_{L}}{\sin t_{L}} \frac{\sin (2 l+1) t_{R}}{\sin t_{R}} \\
\chi^{ \pm}(-e) & = \pm \frac{\sin (2 l+1) t}{\sin t}
\end{aligned}
$$

and the characters of the $((2 l+1,2 r+1))$ rep are given by

$$
\begin{aligned}
& \chi(+e)=\frac{\sin (2 l+1) t_{L}}{\sin t_{L}} \frac{\sin (2 r+1) t_{R}}{\sin t_{R}}+(L \leftrightarrow R) \\
& \chi(-e)=0,
\end{aligned}
$$


where $\chi(+e)$ and $\chi(-e)$ are the characters of the components connected to and disconnected from the identity.

As a manifold, the group is just $\mathrm{SU}(2)^{2} \times \mathbb{Z}_{2}$, and so the normalized group-invariant measure is just one-half of that for $\mathrm{SU}(2)^{2}$ on each connected component. On the component connected to the identity, this reduces to

$$
\frac{2}{\pi^{2}} \int_{0}^{\pi} d t_{L} \sin ^{2} t_{L} \int_{0}^{\pi} d t_{R} \sin ^{2} t_{R}
$$

on class functions, while on the component disconnected from the identity it reduces to

$$
\frac{1}{\pi} \int_{0}^{\pi} d t \sin ^{2} t
$$

One may then easily check that the characters (A.4)-(A.6) are orthonormal with respect to the measure. They are, moreover, a complete set of functions with the given properties. Note that the characters furnish a basis for independent functions on the two disconnected components, as expected.

So far, we have established that the top partners can come in reps formed from the irreps $(2 l+1,2 r+1)$ (without custody of $Z \rightarrow b \bar{b})$ or $(2 l+1,2 l+1)_{ \pm}$and $((2 l+1,2 r+1)$ ) (with custody of $Z \rightarrow b \bar{b}$ ). To establish further constraints on the reps, we need to consider the non-linearly realized group, $G$, and its irreps.

The minimal choice for $G$ (in order to furnish a SM Higgs doublet [5]) is a group locally isomorphic to $\mathrm{SO}(5)$. By similar arguments to those given above for $H$, it suffices to consider the universal cover, viz. $\operatorname{Sp}(2)$. We define $\operatorname{Sp}(n)$ as the group of unitary transformations on $n$-dimensional quaternions, $\mathbb{H}^{n}$, preserving the bilinear $\bar{x} \cdot y$, where we define conjugation of quaternions by $\overline{x_{1}+i x_{2}+j x_{3}+k x_{4}} \equiv x_{1}-i x_{2}-j x_{3}-k x_{4}$. Thus $\mathrm{Sp}(1)$ is isomorphic to $\mathrm{SU}(2)$, and since $\mathrm{Sp}(2) \supset \mathrm{Sp}(1)^{2}$, we have that $\mathrm{Sp}(2) \supset \mathrm{SU}(2)^{2}$. Moreover, the outer automorphism of $\mathrm{SU}(2)^{2}$ that permutes the two $\mathrm{SU}(2) \mathrm{s}$ is an inner automorphism of $\mathrm{Sp}(2)$, so $\mathrm{Sp}(2) \supset \mathrm{SU}(2)^{2} \rtimes \mathbb{Z}_{2}$. Explicitly, the embeddings for the components connected to, and disconnected from, the identity are $\left(\begin{array}{cc}g_{L} & 0 \\ 0 & g_{R}\end{array}\right)$ and $\left(\begin{array}{cc}0 & g_{L} \\ g_{R} & 0\end{array}\right)$, respectively.

We next need to know the irreps of $\operatorname{Sp}(2)$ and how they restrict to the subgroup $\mathrm{SU}(2)^{2} \rtimes \mathbb{Z}_{2}$ (and its subgroup $\mathrm{SU}(2)^{2}$, for models without custodial protection of $Z \rightarrow b \bar{b}$ ). At least for the subgroup $\mathrm{SU}(2)^{2}$, all this can be found in the literature [56]. An irrep of $\operatorname{Sp}(2)$ can be labelled by $(m, k)$, where $m \geq k \geq 0$ and $m$ and $k$ are either both integer or both half-integer, and has dimension $(2 k+1)(2 m+3)(m+k+2)(m-k+1) / 6$. Under restriction to $\mathrm{SU}(2)^{2}$, the irreps $(2 l+1,2 r+1)$ of $\mathrm{SU}(2)^{2}$ arise with multiplicity at most one (since $\mathrm{Sp}(2) / \mathrm{SU}(2)^{2}$ is a symmetric space [57]), and are given by half-integers $0 \leq l, r \leq(k+m) / 2$, with the restriction that for a fixed value of $l, r \in\{|k-l|, \mid k-$ $l \mid+1, \ldots, \min (m-l, k+l)\}$ (and vice versa) for $l \leftrightarrow r$. For $\mathrm{SU}(2)^{2} \rtimes \mathbb{Z}_{2}$, we need only figure out whether the $(2 l+1,2 l+1)$ irreps that appear correspond to $(2 l+1,2 l+1)^{+}$ or $(2 l+1,2 l+1)^{-}$. This can be done by constructing the relevant $\operatorname{Sp}(2)$ irreps as tensor 
products of the fundamental. Indeed, the $\left(\frac{1}{2}, \frac{1}{2}\right)$ irrep has characters

$$
\begin{aligned}
& \chi^{4}(+e)=\cos t_{L}+\cos t_{R}, \\
& \chi^{4}(-e)=0,
\end{aligned}
$$

so the antisymmetric and symmetric parts of its direct product with itself have characters given by $\frac{1}{2}\left(\operatorname{tr}^{2} D \mp \operatorname{tr} D^{2}\right)$, or

$$
\begin{aligned}
& \chi^{[4 \times 4]}(+e)=1+1+\frac{\sin 2 t_{L}}{\sin t_{L}} \frac{\sin 2 t_{R}}{\sin t_{R}} \\
& \chi^{[4 \times 4]}(-e)=1-1-2 \cos t,
\end{aligned}
$$

and

$$
\begin{aligned}
\chi^{(4 \times 4)}(+e) & =4 \cos ^{2} t_{L}+4 \cos ^{2} t_{R}-2+4 \cos t_{L} \cos t_{R}, \\
& =\frac{\sin 3 t_{L}}{\sin t_{L}}+\frac{\sin 3 t_{R}}{\sin t_{R}}+\frac{\sin 2 t_{L}}{\sin t_{L}} \frac{\sin 2 t_{R}}{\sin t_{R}} \\
\chi^{(4 \times 4)}(-e) & =0+2 \cos t,
\end{aligned}
$$

respectively. The first of these shows that $(1,0) \rightarrow(1,1)^{-}+(2,2)^{-}$(and, of course $(0,0) \rightarrow$ $\left.(1,1)^{+}\right)$and the second shows that $(1,1) \rightarrow((1,3)) \oplus(2,2)^{+}$. In general ,we find that the irrep $(m, k)$ restricts to irreps $(2 l+1,2 l+1)^{ \pm}$with \pm given by $(-1)^{(m+k)}$.

These results have four immediate corollaries that that are useful for the classification of top partners: (i) the irrep $(2,2 r+1)$ of $\mathrm{SU}(2)^{2}$ is contained in all irreps with $k \leq r+\frac{1}{2}$ and $m$ sufficiently large, where $k$ (and $m$ ) must be integer or half-integer if $r$ is integer or half-integer, respectively; (ii) for $m$ sufficiently large, these irreps branch to the $\mathrm{SU}(2)^{2}$ irreps $\left(2 l+1,2 r^{\prime}+1\right)$, with $r^{\prime} \in\{|l-k|,|l-k|+1, \ldots l+k\}$; (iii) there are no additional possibilities at small $m$; (iv) only the $(m, 0)$ irreps of $\mathrm{Sp}(2)$ contain singlets of $\mathrm{SU}(2)^{2}$.

Using these results, we see that the possible irreps for top partners depend on whether we wish to have custodial protection of $Z \rightarrow b \bar{b}$ and whether we wish the $t_{R}$ to be fully composite. Custodial protection of $Z \rightarrow b \bar{b}$ (or $Z \rightarrow b_{L} \overline{b_{L}}$, to be precise) is achieved [8] by insisting that the Lagrangian describing the composite sector and the elementary $b_{L}$ be invariant under the subgroup $\mathrm{U}(1)^{2} \rtimes \mathbb{Z}_{2}$. After electroweak symmetry breaking, only the $\mathrm{U}(1) \times \mathbb{Z}_{2}$ (note that it is now a direct product) subgroup is linearly realized, where the $\mathrm{U}(1)$ is the diagonal combination of the 2 original $\mathrm{U}(1) \mathrm{s}$. The irreps of $\mathrm{U}(1)^{2} \rtimes \mathbb{Z}_{2}$ are, in a similar notation to that used for $\mathrm{SU}(2)^{2} \rtimes \mathbb{Z}_{2},(q, q)^{ \pm}$(of dimension 1 ) and $\left(\left(q_{L}, q_{R}\right)\right.$ ) (of dimension 2), where $q, q_{L}$, and $q_{R}$ are the charge $q, q_{L}$, and $q_{R}$ irreps of $\mathrm{U}(1)$. The branching rules under $\mathrm{U}(1)^{2} \rtimes \mathbb{Z}_{2} \rightarrow \mathrm{U}(1) \times \mathbb{Z}_{2}$ are $(q, q)^{ \pm} \rightarrow q$ and $\left(\left(q_{L}, q_{R}\right)\right) \rightarrow\left(q_{L}+q_{R}\right) \oplus\left(q_{L}+q_{R}\right)$. Since U(1) is an unbroken symmetry, $q$ and $q_{L}+q_{R}$ are conserved, and so if the $b_{L}$ carries the $(q, q)^{ \pm}$irrep, its coupling to the $Z$, which is a linear combination of $q$ and the conserved electric charge, is protected. But $b_{L}$ must also transform as a doublet of $\mathrm{SU}(2)_{L}$ and so the only suitable irreps of $\mathrm{SU}(2)^{2} \rtimes \mathbb{Z}_{2}$ are the $(2,2)^{ \pm}$.

Now, a top partner in irrep $\rho_{H}$ of $H$ in such a model must be able to mix with the elementary $Q_{L}$ via the coset representative, and the usual sigma model lore tells us that 
this can happen only if the $Q_{L}$ is assigned to a spurion in a representation $\rho_{G}$ of $G$ that contains $\rho_{H}$ on restriction to $H$.

If we want the $t_{R}$ to be fully composite, it must come in a 1-d irrep of $H$, viz. $(1,1)^{ \pm}$. For if it does not, then there will be additional massless states before EWSB. This too can mix with the $Q_{L}$ via the coset representative only if $\rho_{G}$ contains a $(1,1)^{( \pm)}$.

Thus, if, on the one hand, we desire full $t_{R}$ compositeness, then the top partner must come in an irrep of $H$ that is contained in an irrep of $G$ that contains a $(1,1)^{ \pm}$. The only such irreps of $G$ are of the form $(m, 0)$, with $m \in \mathbb{N}$ and these contain exclusively the irreps $(2 l+1,2 l+1)^{(-1)^{m}}$ with integer $2 l \leq m$. These irreps also all contain a $(2,2)^{ \pm}$, so can feature protection of $Z \rightarrow b \bar{b}$.

If, on the other hand, we desire only protection of $Z \rightarrow b \bar{b}$, then $\rho_{G}$ need only contain a $(2,2)^{ \pm}$and we find in addition the $G$ irreps $(m, 1)$ with $m \in \mathbb{N}$. These contain the $H$ irreps $(2 l+1,2 l+1)^{(-1)^{m+1}}$ with $1 \leq 2 l \leq m$, together with $((2 l+1,2 l+3))$ for $0 \leq 2 l \leq m$.

Finally, if we desire neither $t_{R}$ compositeness nor protection of $Z \rightarrow b \bar{b}$, we need only that the rep $\rho_{G}$ contain a $\left(2,2 r^{\prime}+1\right)$ of $\mathrm{SU}(2)^{2}$, so as to contain the $Q_{L}$. For a suitable $r^{\prime}$, it is then possible to put a top partner in any irrep $(2 l+1,2 r+1)$.

\section{B Details of minimal model without $Z \rightarrow b \bar{b}$ protection}

We define the generators in the $\left(\frac{1}{2}, \frac{1}{2}\right)$ rep of $\operatorname{Sp}(2)$ as

$$
T_{L}^{i}=\frac{1}{\sqrt{2}}\left(\begin{array}{cc}
\sigma^{i} & 0 \\
0 & 0
\end{array}\right) T_{R}^{i}=\frac{1}{\sqrt{2}}\left(\begin{array}{cc}
0 & 0 \\
0 & \sigma^{i}
\end{array}\right) T^{\alpha}=\frac{1}{2}\left[\left(\begin{array}{cc}
0 & \sigma^{i} \\
\sigma^{i} & 0
\end{array}\right),\left(\begin{array}{cc}
0 & -i 1 \\
i 1 & 0
\end{array}\right)\right]
$$

where $T_{L}^{i}, T_{R}^{i}(i=1,2,3)$ are the generators of the $\mathrm{SU}(2)^{2}$ subgroup. The $T^{\alpha}(\alpha=1,2,3,4)$ may be taken in combination with four real fields to form the coset representative

$$
U=\exp \left(\frac{2 i \Pi^{\alpha} T^{\alpha}}{f}\right)=\left(\begin{array}{cc}
1_{2 \times 2} \cos \epsilon & 1_{2 \times 2} \sin \epsilon \\
-1_{2 \times 2} \sin \epsilon & 1_{2 \times 2} \cos \epsilon
\end{array}\right)
$$

where we identify $\Pi^{1,2,3}$ as the Goldstone bosons and $\Pi^{4}$ as the Higgs field of the SM (in the limit $f \rightarrow \infty)$. Thus the last equality gives $U$ in unitary gauge, where $\epsilon \equiv \frac{\left\langle\Pi^{4}\right\rangle}{f}$.

We define the elementary Weyl fermions of the model as

$$
Q_{L}=\left(\begin{array}{llll}
t_{L} & b_{L} & 0 & 0
\end{array}\right)^{T}, t_{R}=\left(\begin{array}{llll}
0 & 0 & t_{R} & 0
\end{array}\right)^{T}, b_{R}=\left(\begin{array}{llll}
0 & 0 & 0 & b_{R}
\end{array}\right)^{T}
$$

and for the top partner

$$
\Psi=\left(\begin{array}{llll}
0 & 0 & T & B
\end{array}\right)^{T} .
$$

In order to construct Lagrangian terms involving the $\Pi$ fields that are invariant under $\operatorname{Sp}(2)$, we consider the quantities

$$
\begin{aligned}
\mathcal{A}_{\mu} & =\frac{1}{\sqrt{2}}\left(g W^{1} T_{L}^{1}+g W^{2} T_{L}^{2}+g W^{3} T_{L}^{3}+g^{\prime} B T_{R}^{3}\right) \\
U^{\dagger}\left(\mathcal{A}_{\mu}+i \partial_{\mu}\right) U & =-d_{\mu}^{i} T^{i}-e_{L \mu}^{i} T_{L}^{i}-e_{R \mu}^{i} T_{R}^{i} .
\end{aligned}
$$


One can show that the $e$ terms transform as a gauge connection under $\mathrm{SU}(2) \times \mathrm{U}(1)$, and may thus form part of the covariant derivative of $\Psi$. The $d$ terms transform linearly under $\mathrm{SU}(2)^{2}$, are comprised of the derivatives of the $\Pi$ fields, and make up the Higgs kinetic terms.

All together, the Lagrangian is

$$
\begin{aligned}
\mathcal{L}= & i \overline{Q_{L}} \not D Q_{L}+i \overline{t_{R}} \not D t_{R}+i \overline{b_{R}} \not D b_{R}+\bar{\Psi}(i \not D-\phi-M) \Psi+\frac{f^{2}}{8} d_{\mu}^{i} d_{i}^{\mu} \\
& -y f \overline{Q_{L}} U \Psi_{R}-y_{R}^{t} f \overline{t_{R}} U \Psi_{L}-y_{R}^{b} f \overline{b_{R}} U \Psi_{L}+\text { h.c }
\end{aligned}
$$

The covariant derivative of the elementary fields is that of the Standard Model, whereas only colour and part of the hypercharge interactions are present in $D_{\mu} \Psi=\partial_{\mu} \Psi-$ $\frac{1}{6} i g^{\prime} B_{\mu} 1_{4 \times 4}-i g_{S} G_{\mu} \cdot{ }^{12}$

To obtain the correct $W$ and $Z$ masses after EWSB, $\epsilon$ is fixed by $v=f \sin \epsilon \cos \epsilon=$ $246 \mathrm{GeV}$.

The Yukawa interactions mix the elementary top and bottom with the $T$ and $B$. Before EWSB $\left(\left\langle\Pi^{4}\right\rangle=0\right)$, the top and bottom remain massless, whereas after $\left(\left\langle\Pi^{4}\right\rangle \neq 0\right)$ the $t, b, T$ and $B$ masses are functions of $y, y_{R}^{t}, y_{R}^{b}, M$ and $f$. Two of these must be fixed to obtain the correct top and bottom masses.

Open Access. This article is distributed under the terms of the Creative Commons Attribution License (CC-BY 4.0), which permits any use, distribution and reproduction in any medium, provided the original author(s) and source are credited.

\section{References}

[1] D.B. Kaplan and H. Georgi, $\mathrm{SU}(2) \times \mathrm{U}(1)$ Breaking by Vacuum Misalignment, Phys. Lett. B 136 (1984) 183 [INSPIRE].

[2] H. Georgi and D.B. Kaplan, Composite Higgs and Custodial SU(2), Phys. Lett. B 145 (1984) 216 [INSPIRE].

[3] M.J. Dugan, H. Georgi and D.B. Kaplan, Anatomy of a Composite Higgs Model, Nucl. Phys. B 254 (1985) 299 [INSPIRE].

[4] R. Contino, Y. Nomura and A. Pomarol, Higgs as a holographic pseudoGoldstone boson, Nucl. Phys. B 671 (2003) 148 [hep-ph/0306259] [INSPIRE].

[5] K. Agashe, R. Contino and A. Pomarol, The minimal composite Higgs model, Nucl. Phys. B 719 (2005) 165 [hep-ph/0412089] [INSPIRE].

[6] G.F. Giudice, C. Grojean, A. Pomarol and R. Rattazzi, The strongly-interacting light Higgs, JHEP 06 (2007) 045 [hep-ph/0703164] [INSPIRE].

[7] P. Sikivie, L. Susskind, M.B. Voloshin and V.I. Zakharov, Isospin Breaking in Technicolor Models, Nucl. Phys. B 173 (1980) 189 [inSPIRE].

\footnotetext{
${ }^{12}$ In order to obtain the correct electric charges for the SM fermions, an extra symmetry $\mathrm{U}(1)_{X}$ is included in both $G$ and $H$, making the linearly realized symmetry group $\mathrm{U}(1)_{X} \times \mathrm{SU}(3)_{c} \times \mathrm{SU}(2)_{L} \times \mathrm{SU}(2)_{R}\left(\rtimes \mathbb{Z}_{2}\right)$. We then gauge a subgroup of this, namely $\mathrm{SU}(3)_{c} \times \mathrm{SU}(2)_{L} \times \mathrm{U}(1)_{Y}$, such that the hypercharge is $Y=T_{R}^{3}+X$. Thus the gauge connections pertaining to colour and part of $\mathrm{U}(1)_{Y}$ appear in $D$, whereas weak isospin and the other part of $\mathrm{U}(1)_{Y}$, these being a subgroup of the $\mathrm{SU}(2)^{2}$ considered above, are present in $e$.
} 
[8] K. Agashe, R. Contino, L. Da Rold and A. Pomarol, A custodial symmetry for $Z b \bar{b}$, Phys. Lett. B 641 (2006) 62 [hep-ph/0605341] [INSPIRE].

[9] D.B. Kaplan, Flavor at SSC energies: a new mechanism for dynamically generated fermion masses, Nucl. Phys. B 365 (1991) 259 [inSPIRE].

[10] B. Gripaios, A. Pomarol, F. Riva and J. Serra, Beyond the Minimal Composite Higgs Model, JHEP 04 (2009) 070 [arXiv: 0902.1483] [INSPIRE].

[11] J. Mrazek et al., The Other Natural Two Higgs Doublet Model, Nucl. Phys. B 853 (2011) 1 [arXiv:1105.5403] [INSPIRE].

[12] A. Falkowski, Pseudo-goldstone Higgs production via gluon fusion, Phys. Rev. D 77 (2008) 055018 [arXiv:0711.0828] [INSPIRE].

[13] I. Low, R. Rattazzi and A. Vichi, Theoretical constraints on the Higgs effective couplings, JHEP 04 (2010) 126 [arXiv:0907.5413] [INSPIRE].

[14] R. Contino, L. Da Rold and A. Pomarol, Light custodians in natural composite Higgs models, Phys. Rev. D 75 (2007) 055014 [hep-ph/0612048] [INSPIRE].

[15] O. Matsedonskyi, G. Panico and A. Wulzer, Light top partners for a light composite Higgs, JHEP 01 (2013) 164 [arXiv:1204.6333] [INSPIRE].

[16] M. Redi and A. Tesi, Implications of a light Higgs in composite models, JHEP 10 (2012) 166 [arXiv: 1205.0232] [INSPIRE].

[17] D. Marzocca, M. Serone and J. Shu, General composite Higgs models, JHEP 08 (2012) 013 [arXiv: 1205.0770] [INSPIRE].

[18] A. Pomarol and F. Riva, The composite Higgs and light resonance connection, JHEP 08 (2012) 135 [arXiv:1205.6434] [INSPIRE].

[19] G. Panico, M. Redi, A. Tesi and A. Wulzer, On the Tuning and the Mass of the Composite Higgs, JHEP 03 (2013) 051 [arXiv: 1210.7114] [INSPIRE].

[20] D. Pappadopulo, A. Thamm and R. Torre, A minimally tuned composite Higgs model from an extra dimension, JHEP 07 (2013) 058 [arXiv: 1303.3062] [INSPIRE].

[21] R. Contino and G. Servant, Discovering the top partners at the LHC using same-sign dilepton final states, JHEP 06 (2008) 026 [arXiv:0801.1679] [INSPIRE].

[22] H. de Sandes and R. Rosenfeld, Search for light custodians in a clean decay channel at the LHC, J. Phys. G 36 (2009) 085001 [arXiv:0811.0984] [INSPIRE].

[23] J.A. Aguilar-Saavedra, Identifying top partners at LHC, JHEP 11 (2009) 030 [arXiv:0907.3155] [INSPIRE].

[24] J. Mrazek and A. Wulzer, A Strong Sector at the LHC: Top Partners in Same-Sign Dileptons, Phys. Rev. D 81 (2010) 075006 [arXiv:0909.3977] [INSPIRE].

[25] G. Dissertori, E. Furlan, F. Moortgat and P. Nef, Discovery potential of top-partners in a realistic composite Higgs model with early LHC data, JHEP 09 (2010) 019 [arXiv: 1005.4414] [INSPIRE].

[26] S. Gopalakrishna, T. Mandal, S. Mitra and R. Tibrewala, LHC Signatures of a Vector-like b', Phys. Rev. D 84 (2011) 055001 [arXiv:1107.4306] [INSPIRE]. 
[27] K. Harigaya, S. Matsumoto, M.M. Nojiri and K. Tobioka, Search for the Top Partner at the LHC using Multi-b-Jet Channels, Phys. Rev. D 86 (2012) 015005 [arXiv:1204.2317] [INSPIRE].

[28] N. Vignaroli, Early discovery of top partners and test of the Higgs nature, Phys. Rev. D 86 (2012) 075017 [arXiv: 1207.0830] [INSPIRE].

[29] A. De Simone, O. Matsedonskyi, R. Rattazzi and A. Wulzer, A First Top Partner Hunter's Guide, JHEP 04 (2013) 004 [arXiv:1211.5663] [INSPIRE].

[30] S. Gopalakrishna, T. Mandal, S. Mitra and G. Moreau, LHC Signatures of Warped-space Vectorlike Quarks, JHEP 08 (2014) 079 [arXiv:1306.2656] [INSPIRE].

[31] A. Banfi, A. Martin and V. Sanz, Probing top-partners in Higgs+jets, JHEP 08 (2014) 053 [arXiv: 1308.4771] [INSPIRE].

[32] A. Azatov, M. Salvarezza, M. Son and M. Spannowsky, Boosting Top Partner Searches in Composite Higgs Models, Phys. Rev. D 89 (2014) 075001 [arXiv: 1308.6601] [INSPIRE].

[33] M. Gillioz, R. Gröber, A. Kapuvari and M. Mühlleitner, Vector-like Bottom Quarks in Composite Higgs Models, JHEP 03 (2014) 037 [arXiv: 1311.4453] [INSPIRE].

[34] O. Matsedonskyi, F. Riva and T. Vantalon, Composite Charge 8/3 Resonances at the LHC, JHEP 04 (2014) 059 [arXiv: 1401.3740] [INSPIRE].

[35] S. Beauceron, G. Cacciapaglia, A. Deandrea and J.D. Ruiz-Alvarez, Fully hadronic decays of a singly produced vector-like top partner at the LHC, arXiv:1401.5979 [INSPIRE].

[36] N.G. Ortiz, J. Ferrando, D. Kar and M. Spannowsky, Reconstructing singly produced top partners in decays to $\mathbf{W b}$, arXiv: 1403.7490 [INSPIRE].

[37] D. Barducci et al., Model Independent Framework for Analysis of Scenarios with Multiple Heavy Extra Quarks, arXiv:1405.0737 [INSPIRE].

[38] M. Endo, K. Hamaguchi, K. Ishikawa and M. Stoll, Reconstruction of Vector-like Top Partner from Fully Hadronic Final States, arXiv: 1405.2677 [INSPIRE].

[39] B. Gripaios, Composite Leptoquarks at the LHC, JHEP 02 (2010) 045 [arXiv:0910.1789] [INSPIRE].

[40] B. Gripaios, A. Papaefstathiou, K. Sakurai and B. Webber, Searching for third-generation composite leptoquarks at the LHC, JHEP 01 (2011) 156 [arXiv:1010.3962] [INSPIRE].

[41] S.R. Coleman, J. Wess and B. Zumino, Structure of phenomenological Lagrangians. 1., Phys. Rev. 177 (1969) 2239 [inSPIRE].

[42] CMS collaboration, Search for top-quark partners with charge $5 / 3$ in the same-sign dilepton final state, Phys. Rev. Lett. 112 (2014) 171801 [arXiv:1312.2391] [INSPIRE].

[43] ATLAS collaboration, Search for anomalous production of events with same-sign dileptons and $b$ jets in $14.3 \mathrm{fb}^{-1}$ of pp collisions at $\sqrt{s}=8 \mathrm{TeV}$ with the ATLAS detector, ATLAS-CONF-2013-051 (2013).

[44] A. Alloul, N.D. Christensen, C. Degrande, C. Duhr and B. Fuks, FeynRules 2.0 - A complete toolbox for tree-level phenomenology, Comput. Phys. Commun. 185 (2014) 2250 [arXiv: 1310.1921] [INSPIRE].

[45] F. Maltoni and T. Stelzer, MadEvent: automatic event generation with MadGraph, JHEP 02 (2003) 027 [hep-ph/0208156] [INSPIRE]. 
[46] T. Sjöstrand, S. Mrenna and P.Z. Skands, PYTHIA 6.4 Physics and Manual, JHEP 05 (2006) 026 [hep-ph/0603175] [INSPIRE].

[47] DELPHES 3 collaboration, J. de Favereau et al., DELPHES 3, A modular framework for fast simulation of a generic collider experiment, JHEP 02 (2014) 057 [arXiv:1307.6346] [INSPIRE].

[48] A.L. Read, Presentation of search results: The $C L_{s}$ technique, J. Phys. G 28 (2002) 2693 [INSPIRE].

[49] ATLAS collaboration, Search for direct third-generation squark pair production in final states with missing transverse momentum and two b-jets in $\sqrt{s}=8 \mathrm{TeV} p p$ collisions with the ATLAS detector, JHEP 10 (2013) 189 [arXiv:1308.2631] [INSPIRE].

[50] ATLAS collaboration, Search for direct top squark pair production in final states with one isolated lepton, jets and missing transverse momentum in sqrts $=8, \mathrm{TeV}$ pp collisions using $21 \mathrm{fb}^{-1}$ of ATLAS data, ATLAS-CONF-2013-037 (2013).

[51] CMS collaboration, A search for new physics in events with high jet and b-tagged jet multiplicities and one lepton, CMS-PAS-SUS-12-015 (2013).

[52] CMS collaboration, Inclusive search for a vector-like T quark with charge $\frac{2}{3}$ in pp collisions at $\sqrt{s}=8 \mathrm{TeV}$, Phys. Lett. B 729 (2014) 149 [arXiv:1311.7667] [InSPIRE].

[53] ATLAS collaboration, Search for pair production of heavy top-like quarks decaying to a high- $p_{\mathrm{T}} W$ boson and $a b$ quark in the lepton plus jets final state in pp collisions at $\sqrt{s}=8 \mathrm{TeV}$ with the ATLAS detector, ATLAS-CONF-2013-060 (2013).

[54] CMS Collaboration, Search for Vector-Like b' Pair Production with Multilepton Final States in pp collisions at $\sqrt{s}=8 \mathrm{TeV}$, CMS-PAS-B2G-13-003 (2013).

[55] T. Broecker and T. tom Dieck, Representations of Compact Lie Groups, Springer, Berlin Germany (1985).

[56] N. Kemmer, D.L. Pursey and S.A. Williams, Irreducible representations of the fivedimensional rotation group. I, J. Math. Phys. 9 (1968) 1224.

[57] S. Helgason, Graduate Studies in Mathematics. Vol. 34: Differential Geometry, Lie Groups, and Symmetric Spaces, American Mathematical Society, Providence U.S.A. (2001). 